\title{
Use of Acellular Fish Skin for Dura Repair in an Ovine Model: A Pilot Study
}

\author{
Hilmar Kjartansson 1,2, Ingvar Hakon Olafsson'2, Sigurbergur Karason2 ${ }^{2}$, Hjalti Thorisson 2,3, \\ Baldur Tumi Baldursson 1,2, Eggert Gunnarsson", Einar Jorundsson", \\ Gudmundur Fertram Sigurjonsson 1 \\ ${ }^{1}$ Kerecis Limited, Reykjavik, Iceland \\ ${ }^{2}$ The National University Hospital of Iceland, Reykjavik, Iceland \\ ${ }^{3}$ Domus Radiology Center, Reykjavik, Iceland \\ ${ }^{4}$ The Institute for Experimental Pathology, University of Iceland, Reykjavik, Iceland \\ Email: hkj@kerecis.com
}

Received 1 September 2015; accepted 26 October 2015; published 29 October 2015

Copyright (C) 2015 by authors and Scientific Research Publishing Inc.

This work is licensed under the Creative Commons Attribution International License (CC BY).

http://creativecommons.org/licenses/by/4.0/

(c) (i) Open Access

\section{Abstract}

Recently the use of biologic materials as dura mater repair patches has been increasing. The purpose of this study is to assess the basis for efficacy and safety of using a novel fish derived acellular dermis (Kerecis Omega3 Dura ${ }^{\mathrm{TM}}$ ). In an ovine model a craniotomy under general anaesthesia was performed. A defect was produced in the dural covering of approximately $1 \times 2 \mathrm{~cm}$ and closed with an onlay technique, with Kerecis Omega3 Dura. The bone defect was covered with the bony flap and the overlying tissues closed in layers. At 2, 5, 8 and 11 weeks the sheep underwent MRI scanning followed by euthanasia, necropsy and histological assessment. MRI images taken at 2, 5, 8 and 11 weeks showed initially moderate inflammatory response, which diminished over time, and at 11 weeks no evidence of inflammation existed. There was evidence of cerebrospinal fluid leakage at no time point. Necropsy revealed some adhesions at 5 and 8 weeks, in particular at 5 weeks, but at 11 weeks there were no adhesions found. From 2 - 11 weeks, there was evidence of initially an inflammatory reaction followed by neodura formation at the defect site through cellular ingrowth and remodeling of the acellular fish skin. Histology showed a histiocytic foreign body reaction initially that subsided over time. As early as 8 weeks there was evidence of neodura formation and by 11 weeks there was a minimal inflammatory response with an intact neodura formed. In this pilot study the Kerecis Omega3 Dura patches performed in a safe and efficacious manner. This new material needs to be fully assessed and compared with other products that are currently on the market in a larger scale animal study.

\section{Keywords}

Duraplasty, Dura Repair, Piscine Acellular Dermal Graft, Neurosurgery, Dura Mater, Fish Skin Graft, Acellular Fish Skin, Craniotomy, Ovine Model, Dural Defect 


\section{Introduction}

All commercially available tissue based or tissue derived materials used for implantation to date are derived from mammalian sources. Biologic tissue based materials are normally referred to as Acellular Dermal Matrices (ADM's) and Extra Cellular Matrices (ECM's). Biologic tissue derived materials are normally referred to as collagen scaffolds. Tissue based materials are intact tissues where cells and fats have been washed away with detergents because of disease transmission risk. As no disease transmission risk exists in the use of fish skin, fats are not washed away during processing and hence the materials are referred to as acellular fish skin. Tissue derived materials are denatured protein polymers (normally collagen) that have been extracted from tissue through hydrolysis.

The use of tissue based and tissue derived materials within various bioengineering fields has grown rapidly due to their excellent biocompatibility, low antigenicity, high biodegradability and good mechanical, haemostatic and cell-binding properties. Acellular fish skin has the potential for use in a variety of clinical scenarios such as management of wounds, oral cavity wound repair, dura mater repair, hernia surgery, treatment of burns and foreseeable for tissue augmentation in plastic surgery such as breast reconstruction and abdominal wall reconstruction. Prior to human clinical use, it is important to evaluate how the acellular fish skin behaves in vivo compared to mammalian derived products. It is also important to document the tissue reaction and compare to results in the literature as well as to discover potential adverse reactions.

Biologic dura mater implants possess a proven efficacy record, both in established animal models as well as in clinical use [1]. Biologic products have been assessed in a variety of neurosurgical procedures, proving to be safe and effective. An example of such an assessment is a study with an onlay suture-free technique, where the results demonstrated that operating theatre time was minimized and that no secondary cerebrospinal fluid (CSF) leaks were observed [2]-[6].

The requirements for an ideal dura mater substitute are: no induction of an immune or inflammatory response and no neurotoxicity. The material should rapidly resorb and be replaced with the body's own connective tissue so that neodura is formed. The material should not cause significant adhesions or abundance of fibrosis. The material needs to be resistant to tearing, watertight as well as being easy to handle and apply. Other important criteria are viral and prion safety and economical attractiveness.

Some form of dura mater defect patching is required in association with almost $30 \%$ of all craniotomies [7]. The need for dura mater patching materials with superb clinical usability is therefore of great practical significance.

In recent years, a firm relationship has been determined between iatrogenic Creutzfeld-Jakob disease and human cadaveric biologic dura substitutes [8] [9]. The impossibility of completely ruling out this significant risk has triggered the ongoing search for new risk free dura substitutes, including various types of dural sealants, dura substitutes and growth factors [10].

Fish does not possess prions or viruses transmittable to humans. Acellular fish skin grafts are therefore an exciting alternative to mammalian biologic materials. Regulatory authorities require mammalian xenograft product to be from specially raised flocks of animals in BSE free countries. Also genetically modified pigs are normally used where the gene encoding alpha-gal has been removed. Alpha-gal is the main allergic component related to human use of porcine xenografts.

\section{Material and Methods}

\subsection{Experimental Animals}

The study was performed on five adult domestic sheep of Icelandic breed based on an approval from the approval from the ethical committee on animal and laboratory research. The animals were kept in an animal pen at the Institute for Experimental Pathology, University of Iceland, Keldur.

The literature contains the results of multiple dura mater studies in various animal models. The most common small animal models are rabbit or canine [1] [11] [12]. Large animal models include both porcine and ovine models. Both the efficacy and safety of biologic patches in the repair of dura defects has been evaluated in such models [13] [14]. In the case of this study, a large animal model was considered to better simulate the surgical procedures in humans resulting in the use of an ovine model. 


\subsection{Allocation to Study Groups}

The five sheep in the study were allocated randomly between the time periods of 2, 4, 6, 8 and 10 weeks for neuro imaging, followed by necropsy and histological analysis.

\subsection{Investigated Products}

Kerecis Omega3 Dura is acellular fish skin from North Atlantic cod fish (Gadus morhua). The material is produced by immediately harvesting fish skin from the caught fish. The skin is then de-scaled, followed by gentle acelleration where the skin's cells are removed without interrupting the natural structure of the skin. Differently from processing of mammalian tissues no harsh detergents are used during processing leaving the fatty component of the tissue largely undisturbed. Finally water is removed from the skin with freeze drying, the material packed and sterilized.

\subsection{Anaesthesia and Euthanasia}

Anaesthesation and euthanisation was performed in the following manner:

As with all surgical procedures there is the possibility of pain postoperatively. In order to minimize any suffering to the animals appropriate measures were taken, prior to, during and after the operation by using the most suitable pharmaceuticals as described below. The perioperative process was performed through a cooperation between a veterinarian and a medical anaesthesiologist.

Premedication: Xylazin (Chanazin 10\%, Chanelle Pharmaceuticals Manufacturing Ltd., Ireland) 0.5 - $1 \mathrm{ml} / 50$ kg i.v. $+1 \mathrm{ml}$ Atropin s.c.

Anaesthesia: Ketamin (Ketador Vet 100 mg/ml, Richter Pharma AG, Austria) 100 mg/ml: 1 ml/50ml. i.v.

When the animal became drowsy, an intravenous catheter was inserted in the right jugular vein.

Saturation and pulse was monitored by attaching a pulseoxymeter onto an ear.

Endotraacheal intubation was facilitated with i.v. propofol boluses.

General anaesthesia was accomplished using a conventional anaesthesia machine for humans with Isofluran on spontaneously breathing intubated animals in the prone position.

Isoflurane and oxygen concentration in inspiratory air and $\mathrm{CO}_{2}$ in expiratory air was monitored continuously.

No muscle relaxants were given, allowing the animals to breathe spontaneously and to move if experiencing pain, allowing easy monitioring of the anaesthesia depth.

Flunixin (Finadyne vet $50 \mathrm{mg} / \mathrm{ml}$, Intervet International BV, The Netherlands) $1 \mathrm{ml} / 50 \mathrm{~kg}$ s.c. post operative pain relieve was provided daily. Duragesic depot plaster was provided one day before operation as well as on the day of operation and then every second day up to one week after operation.

Peri and post-operative, phenoxymethyl-penicillin was given for 3 days.

During neuroimaging, the animals received the same premedication as described above, then an i.v. jugular catheter was inserted and the animal sedated with intermittent i.v. propofol boluses, allowing spontanoues breathing, under continuos observation.

Euthanasia: Euthasol vet $400 \mathrm{mg} / \mathrm{ml}$ i.v.

\subsection{Surgical Technique}

All the animals had a right craniotomy over frontoparietal area performed as follows: The wool on the head was shaved, followed by a chlorhexidine wash (0.5\%). Sterile drapes were applied for the surgical field. Local anaesthesia for the operation and post-operative pain was achieved with Bupivacaine $5 \mathrm{mg} / \mathrm{ml} \&$ adrenaline 5 microgr $/ \mathrm{ml}$ mixture in scalp. The skin was incised to the right of the midline and a right sided craniotomy performed. The bone was then removed with great care, in order to prevent injury to the arachnoidea mater. An incision into the dura was made, creating a defect of approximately $1 \times 2 \mathrm{~cm}$. The defect was covered with Kerecis Omega3 Dura as an onlay, tucking the ends underneath the circular defect in the skull. The bone defect was then closed with the excised bone flap. The galea was closed using absorbable sutures and the closure of the scalp was achieved with interrupted sutures.

\subsection{Postoperative Observation of the Animals}

The animals were returned to their pen approximately 30 minutes after extubation. They were checked hourly 
for any signs of inflammation or neurological abnormality in the first 4 hours and then daily after that.

Post operative pain relieve was achieved with Finadyne vet (flunixin) $1 \mathrm{ml} / 50 \mathrm{~kg}$ s.c daily and Duragesic depot plaster once before the day of operation and then every second day up to one week after the operation.

\subsection{Neuroimaging}

At preplanned time intervals the animals were brought in for Magnetic resonance imaging (MRI) with and without gadolinium contrast enhancement, to look for; evidence of cerebral inflammation or damage, evidence of CSF leak and graft visualization.

\subsection{Sampling}

On the day following neuroimaging, individual animals were sacrificed with euthanasia. Euthanasia was performed by an experienced veterinarian with Euthasol vet $400 \mathrm{mg} / \mathrm{ml}$ (pentobarbitol). Scheduled time points were after 2, 4, 6, 8 and 10 weeks post operation for one animal at each timepoint.

The euthanized animals had their heads surgically split, sagitally slightly to the left of the midline. Gentle dissection was the done to remove the rest of the left side of the cerebrum. The implant side was then examined for any evidence of macroscopic adhesions between the surface of the cerebrum to the implant material, followed by gentle blunt dissection. The surface of the brain was inspected and then fixed in formaldehyde. The dura and graft material was then dissected from the interior of the skull and fixed in formaldehyde.

\subsection{Histological Methods}

Tissue sections were stained with H\&E and Movat pentachrome (MP), prior to histometric scoring for acute and chronic inflammation as well as eosinophilic infiltration. Sections were stained in a standard manner with hematoxylin and eosin (HE). Modified Movat's pentachrome staining was performed in the following manner:

Briefly, sections were stained with $1 \%$ alcian blue solution for $20 \mathrm{~min}$, washed briefly in water and placed in preheated ammonium hydroxide solution $\left(56^{\circ} \mathrm{C}\right)$ for $10 \mathrm{~min}$ and washed for $5 \mathrm{~min}$ under cold running water. The slides were placed in orcein-verhoeff working solution for $7 \mathrm{~min}$ and washed for $5 \mathrm{~min}$ under cold running water, then stained with woodstain scarlet-acid fuchsin working solution, followed by differentiation in $0.5 \%$ acetic acid and incubated with 5\% phosphotungstic acid for 5 - $10 \mathrm{~min}$. The slides were transferred directly into $1 \%$ acetic acid for $5 \mathrm{~min}$, and then rinsed in three changes of absolute alcohol. Finally the slides were placed in alcoholic safran solution for 8 minutes, rinsed in three changes of absolute alcohol, cleared in xylene and mounted.

The objective of histological assessment was to:

- Describe and quantify the inflammatory reaction in the graft site (epidural, subdural, transitional zone between dura and graft).

- Evaluate the extent of foreign body reaction.

- Evaluate the degree of connective tissue organization of the graft.

- Evaluate changes to the cerebral cortex (inflammation and necrosis).

\section{Results}

\subsection{Intraoperative and Postoperative Course}

Anaesthesia, surgery and the postoperative follow up was uneventful in all but one animal. The first animal operated on was difficult to intubate and ended up having a cricothyroidotomy performed for safe airway management. The animal had a lengthy general anaesthetic and operating time, however appeared well later in the day but then died overnight. Necropsy performed showed no evidence of an acute reaction at the graft site but some intraoperative bruising had been noted. The death was deemed to be a result of the complicated operation. No compromise of the airway was though noted. Due to the reduced number of animals, the chosen time points were changed to achieve an improved scope to reflect the same overall time period, with one animal being sacrificed at 2, 5, 8 and 11 weeks respectively.

There was minimal intraoperative bleeding and in most cases from small blood vessels of the dura mater. Intraoperative hemostasis was unproblematic. There was suspicion of a possible minor contusion intraopera- 
tively on the first sheep.

None of the animals displayed neurological abnormalities during postoperative follow up. Likewise, none of the animals displayed signs of inflammation, cerebrospinal fluid leakage or impaired wound healing. No complications related to the anaesthesia were noted.

\subsection{Necropsy}

At 2 weeks the surgical wound had healed well with no evidence of inflammatory changes. The surface of the brain appears normal, with $2 \mathrm{~mm}$ adhesion between brain and overlying dura repair. Mild intrusion of bony flap as seen on MRI. Adhesion bluntly dissected without any problems. No erythema or inflammation noted on cerebral surface (Figure 1). Right side of brain removed enbloc and put in formaldehyde prior to fixation and histology. Interior surface of dura shows the defect has closed and the dural patch is not visible through it. Appears as a normal dura. On the outer surface the fish skin dura patch is visible and intact. Shows evidence of integration into surrounding dura. Extradural tissues look benign with minor granulation tissue noted. The dura patch with the surround dura is excised enbloc and placed in formaldehyde.

At 5 weeks the wound is well healed. There is evidence of protrusion of the bony flap by approximately $4 \mathrm{~mm}$. Underneath the dura patch in that area, there is evidence of adhesion over an area of 5 - $10 \mathrm{~mm}$ (Figure 2). The adhesions were bluntly dissected and on the surface of the cerebrum there was a visible yellowish discoloration considered to be from traces of hemosiderin. The brain was fixed in formaldehyde. The fish skin patch and surrounding dura show evidence of integration and were excised enbloc and placed in formaldehyde.

At 8 weeks the external wound is well healed. There is approximately $2 \mathrm{~mm}$ intrusion of the bony flap and underneath that, a minor $2 \mathrm{~mm}$ adhesion, that was easily bluntly dissected from the underlying brain. The cerebral surface appears normal. The fish skin patch adheres to the overlying bone and was bluntly dissected away (Figure 3). The outer dural surface shows good integration of dura and the dura patch, which is still visible and the area is slightly thinner than the normal dura and slightly oedematous. Brain and dura patch with surrounding dura fixed in formaldehyde.

At 11 weeks the external wound is fully healed. The bony flap sits well in the cranial defect with no intrusion. There is no evidence of any adhesion and the cerebral surface appears normal (Figure 4). The fish skin patch is slightly visible and the area shows excellent integration into surround dura. Specimens fixed in formaldehyde as above.

\subsection{Neuroimaging}

One day prior to necropsy, each individual sheep underwent a Magnetic Resonance Imaging (MRI) scan. The following MRI sequences were performed on each individual: Axial and coronal T2, Axial FLAIR, Axial diffusion weighted imaging (DWI), Sagital T1 SPGR (3D series) before and after administration of intravenous gadolinium contrast (dose approximately $1 \mathrm{mg} / \mathrm{kg}$ ).

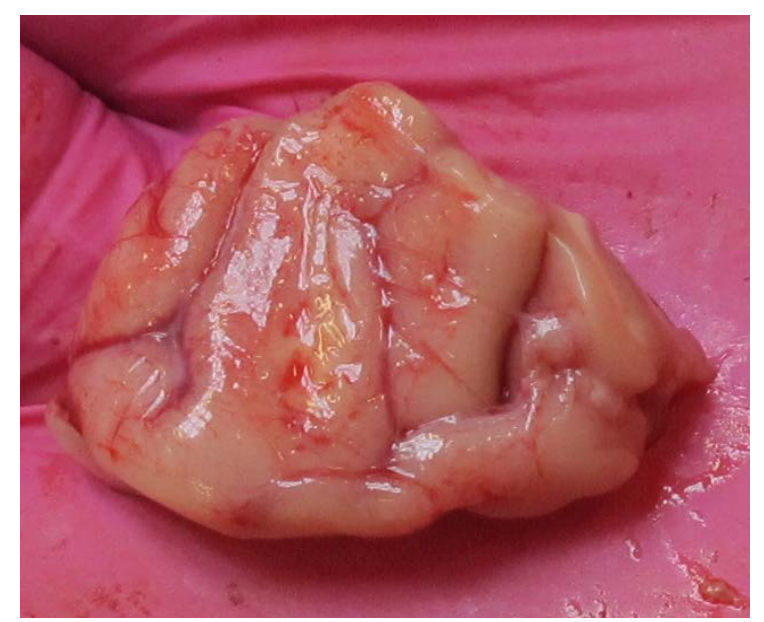

Figure 1. At 2 weeks, minimal evidence of inflammatory changes on cerebral surfaces. 


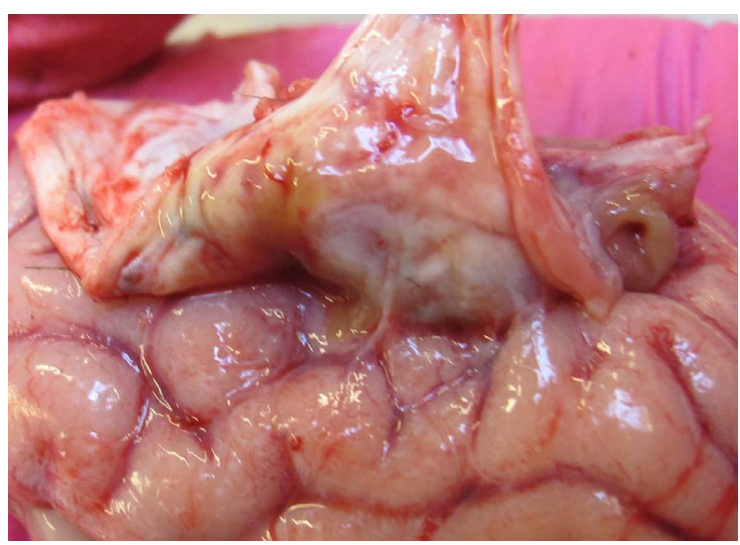

Figure 2. Adhesions noted under the bony intrusion at 5 weeks.

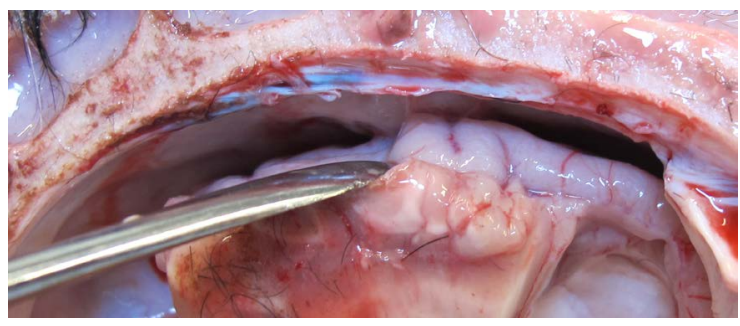

Figure 3. At 8 weeks, minimal adhesion is visible directly under the bony intrusion.

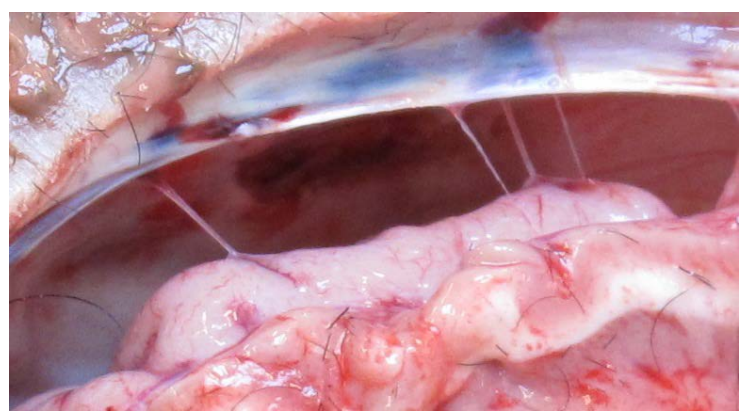

Figure 4. At 11 weeks there is no evidence of adhesion underneath the dura repair (the dark area shown with the arrow).

General description: none of the subjects showed evidence of subdural fluid collection, cerebral inflammatory changes, enlargement of the ventricular systems nor any parenchymal abnormality (with the exception of one subject that showed very minimal intraparenchymal hemorrhage believed to be post-operative in nature). The operative zone was noted to be in the frontoparietal area slightly to the right of the midline.

At 2 weeks there is evidence of post-operative inflammation within the surgical bed with an extradural fluid collection with a maximum thickness of $7 \mathrm{~mm}$. Extracalvarial fluid was also identified, with a maximum thickness of $3-4 \mathrm{~mm}$. There is an area of post contrast enhancement of $3 \mathrm{~mm}$ thickness adjacent to the brain. The fish skin patch was not identifiable (Figure 5).

At 5 weeks there is no evidence of any fluid accumulation. There is mild contrast enhancement that was concluded to be of the magnitude expected after a craniotomy and dura compromise. On one T1 image there is evidence of a tiny hairpin $3 \mathrm{~mm}$ intracerebral focus of hemorrhage adjacent to an incursion of the bony flap, that was replaced postoperatively. The radiologists conclusion was that the hemorrhage is the result of secondary pressure from the bony flap and thus a post-operative finding (Figure 5). 


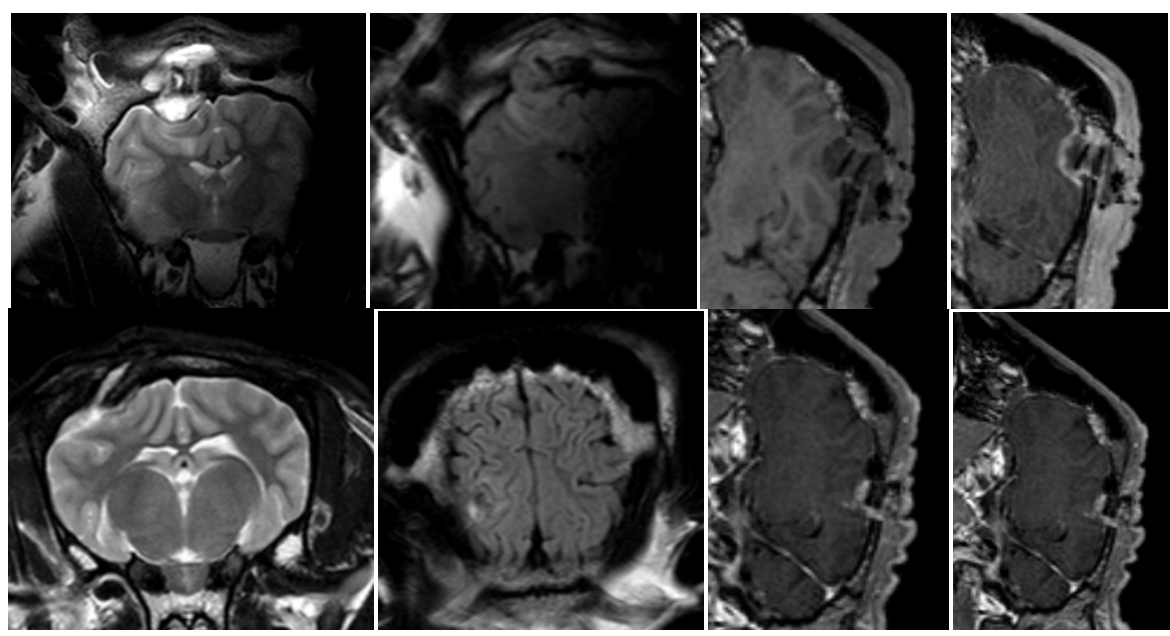

Figure 5. MRI images at weeks 2 (above) and 5 (lower). From left to right: 1) the coronal, 2) axial and 3) sagital planes followed by 4) a saggital view with gadolinium contrast enhancement.

At 8 weeks there is a minimally increased signal involving the operative bed, but no abnormal signal of the cerebrum on T2 or FLAIR images. No fluid collection and only minor contrast enhancement noted and considered to be within normal limits after a craniotomy and dura operation. The bony flap is "tilted" within the craniotomy defect, with some incursion into the calvarium, but no underlying inflammatory changes of note (Figure 6).

At 11 weeks the defect site would not have been noticeable of not for the overlying bony defect. Only very minimal contrast enhancement is noted in the region of the dura, despite recent surgery (Figure 6).

\subsection{Macroscopy and Histology}

Macroscopically, all the animals displayed reliable closure of the dura defect, with no graft rejection or reactions. Small adhesions between the implant and cortical structures developed in most cases, probably due to tiny injuries to the arachnoid during surgery.

Two weeks postoperatively, the graft site showed extensive neutrophilic inflammation enclosing and infiltrating the fish skin graft, with a surrounding layer of mononuclear leukocytes and a few multinuclear giant cells. The subarachnoid space was closed by infiltration of lymphocytes and macrophages. (Figure 7 and Figure 8)

After five weeks, inflammatory changes in the fish skin graft had regressed, but a continuous layer of multinuclear giant cells enclosed the graft, gradually replacing the graft from the periphery. The layer of giant cells was surrounded by an intense infiltration of lymphocytes. The inflammatory adhesion of the leptomeninges was still present (Figure 9 and Figure 10).

Eight weeks after the operation, inflammatory processes in the neodura had regressed further. MP-stained slides showed neogenesis of tissue and emerging new blood vessels (angioneogenesis). The subarachnoid space was again evident in the periphery, but directly below the graft site mononuclear inflammatory infiltrates are still evident (Figure 11 and Figure 12).

After 11 weeks, a further regression of the cellular inflammatory response was evident, with only clusters of lymphocytic and monocytic nodular infiltrates still visible and re-opening of the subarachnoid space (Figure 13 and Figure 14).

The results are summarized in Table 1.

The histological examination concludes that the acellular fish skin graft acts as host for cellular ingrowth and allows for neodura formation. This is evident on the graft sites in all the sacrificed animals.

\section{Discussion}

\subsection{Necropsy}

The macroscopic findings at necropsy are promising. Most of the documented changes are in-line with expecta- 


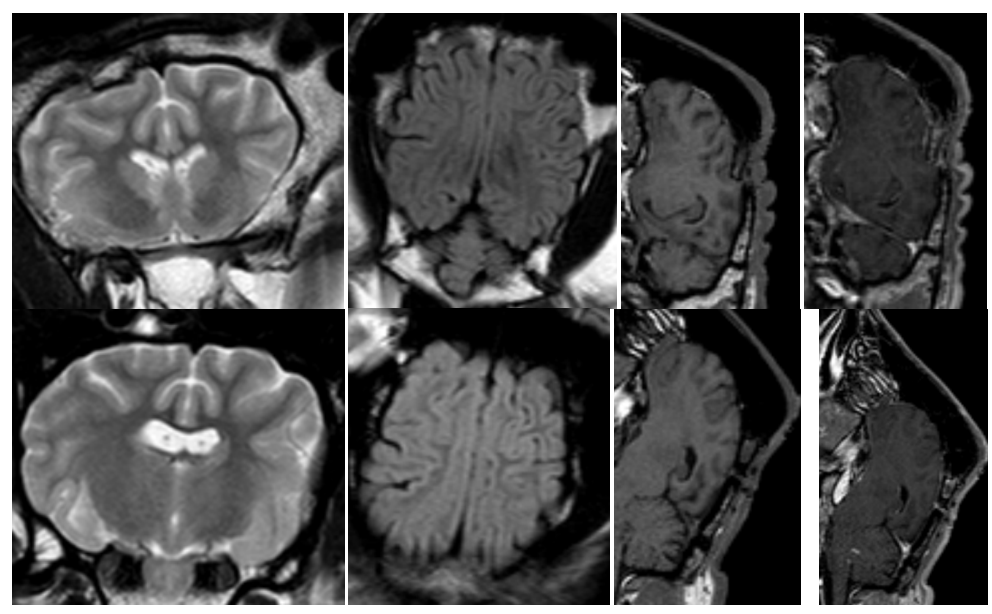

Figure 6. MRIs taken at weeks 8 (above) and 11 (lower). From left to right, 1) the coronal, 2) axial and 3) saggital planes followed by 4) a sagital view with gdolinium contrast enhancement.

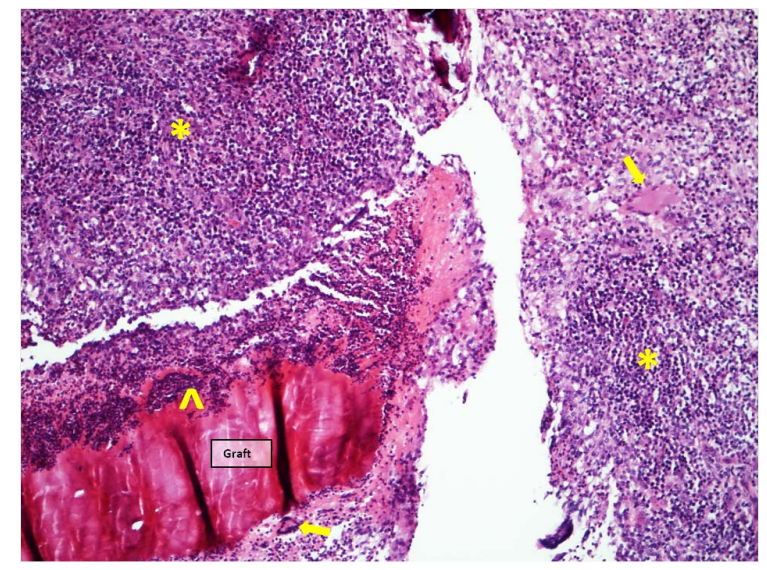

Figure 7. Graft 2 weeks postoperatively infiltrated by neutrophils $(\wedge)$ and surrounded by a layer of mononuclear leukocytes $(*)$ with few multinuclear giant cells (arrows) (HE-staining, $10 \times$ objective).

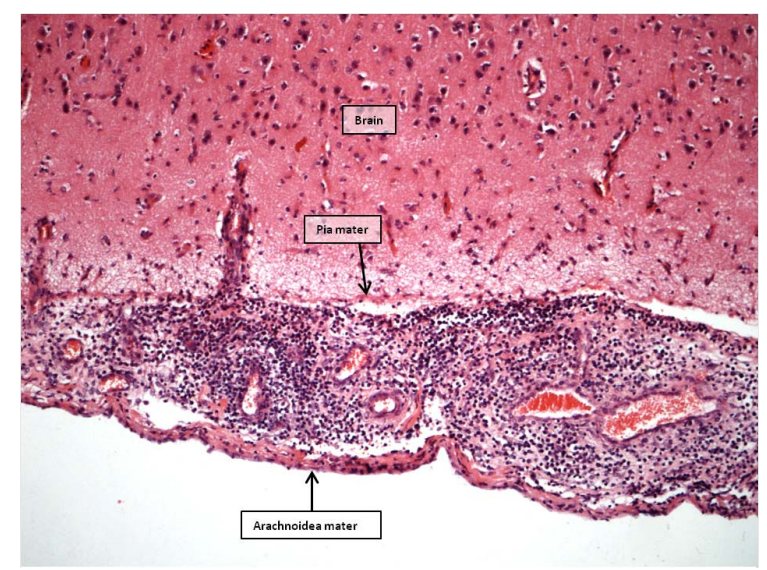

Figure 8. Brain 2 weeks postoperatively. Subarachnoid space filled with mononuclear leukocytes (HE-staining, $10 \times$ objective). 


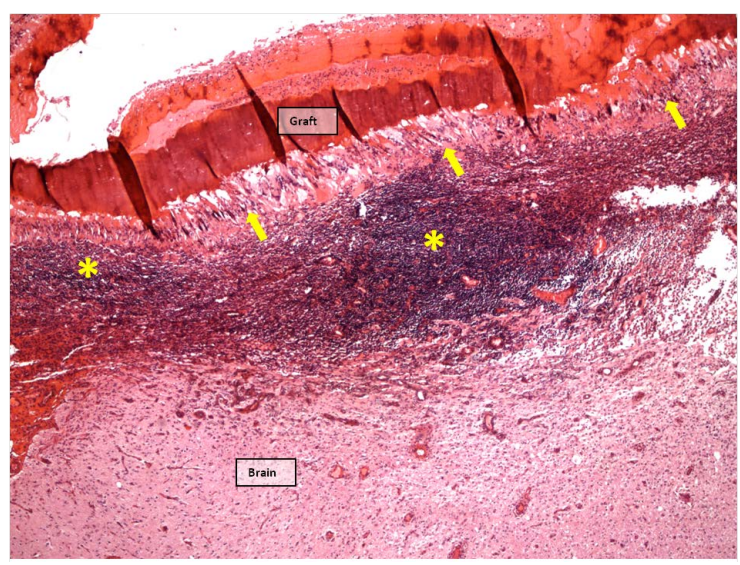

Figure 9. Graft 5 weeks postoperatively surrounded by multinuclear giant cells (arrows). A layer of mononuclear leukocytes $(*)$ infiltrate subarachnoid space through the underlying pia mater (HE-staining, $4 \times$ objective).

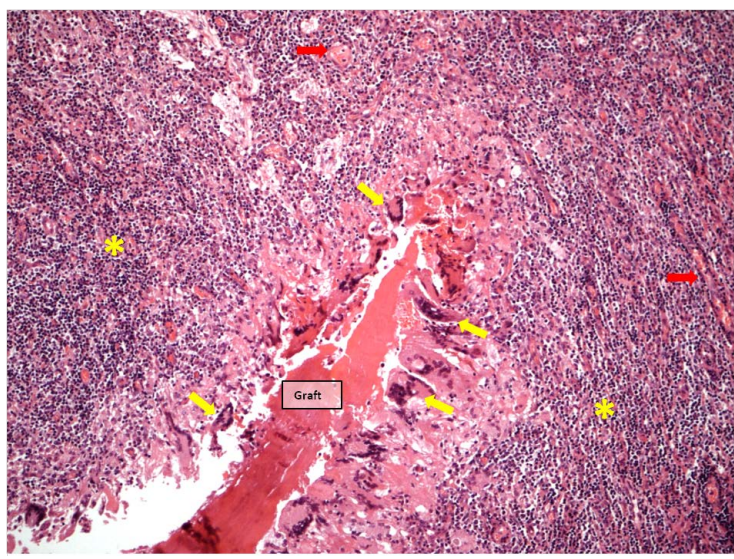

Figure 10. Graft 5 weeks postoperatively surrounded by multinuclear giant cells (yellow arrows) and mononuclear infiltrates $(*)$. Early organization and angioneogenesis (red arrows) (MP-staining, $4 \times$ objective).

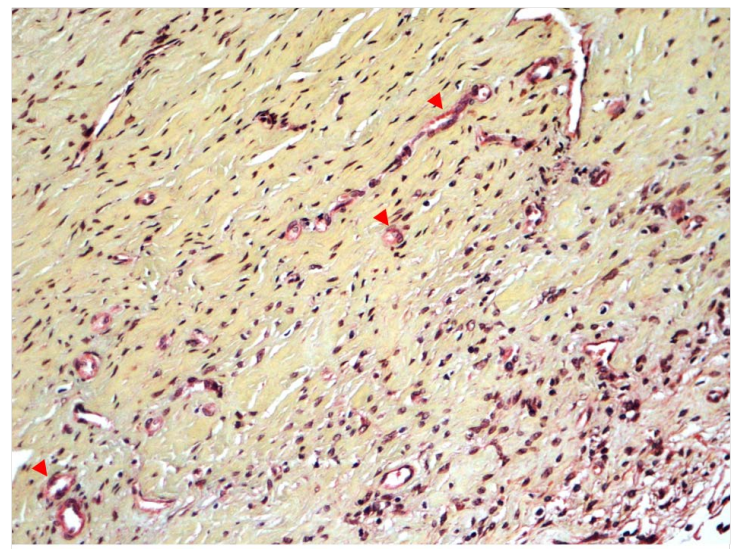

Figure 11. Graft 8 weeks postoperatively showing dura with dense collagen fibers (yellow-green), fibroblasts and newly formed capillaries (arrowheads). Mild perivasular mononuclear inflammation (MP-staining, 20× objective). 


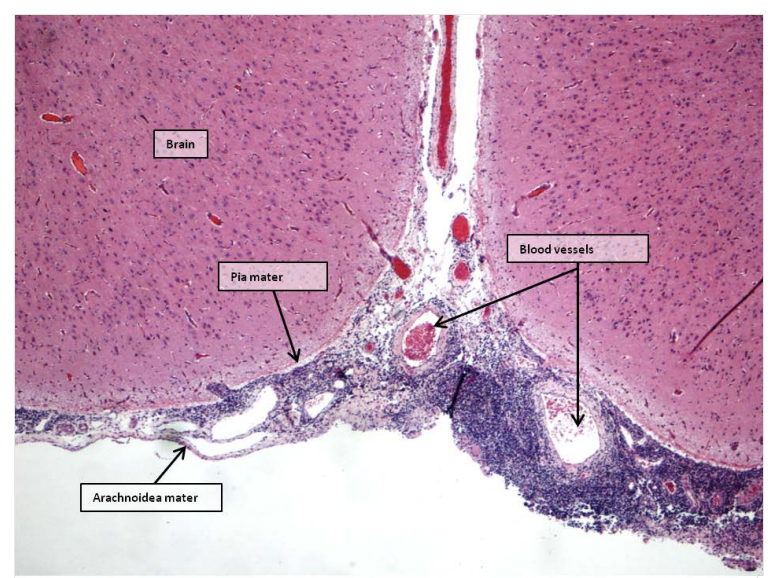

Figure 12. Brain 8 weeks postoperatively. Subarachnoid space partly filled with mononuclear leukocytes (*) (HEstaining, $4 \times$ objective).

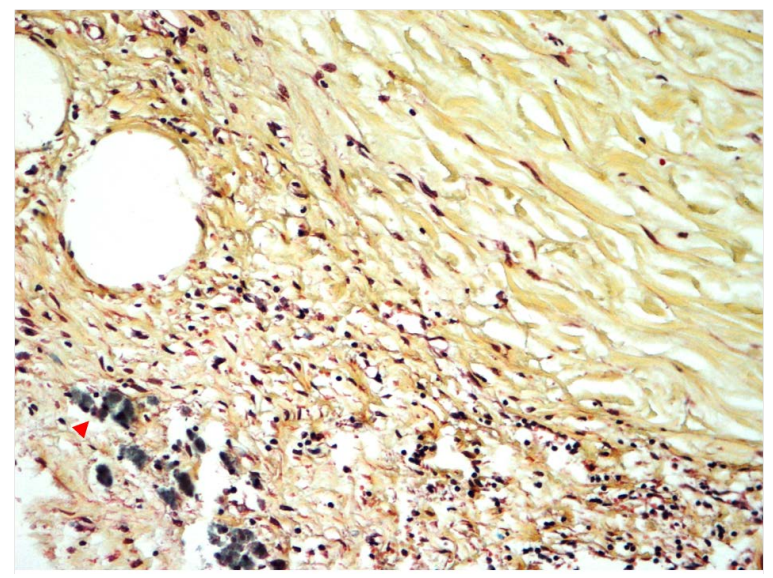

Figure 13. Graft 11 weeks postoperatively showing dura with dense collagen fibers (yellow-green), mild perivasular mononuclear inflammation and hemosiderin laden macrophages (red arrowheads) (MP-staining, 20× objective).

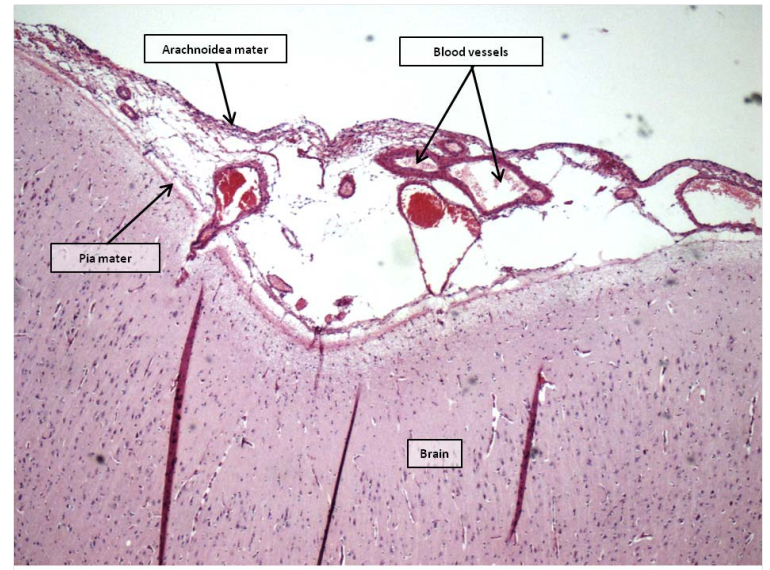

Figure 14. Brain 11 weeks postoperatively. Open subarachnoid space with few scatterd lymphocytes (HE-staining, $4 \times$ objective). 
Table 1. Quantification of inflammatory response, connective tissue organization and extent of foreign body reaction in graft sites and subdural and epidural space.

\begin{tabular}{|c|c|c|c|c|c|}
\hline \multirow{2}{*}{ Time post op. } & \multirow{2}{*}{ Animal nr } & \multicolumn{3}{|c|}{ Graft, sub-epidural space } & \multirow{2}{*}{$\begin{array}{c}\text { Subarachnoid space } \\
\text { Inflammation }\end{array}$} \\
\hline & & Inflammation & Organization & Foreign body reaction & \\
\hline Wk 2 & 31 & +++ & - & + & +++ \\
\hline Wk 5 & 36 & ++ & + & ++ & ++ \\
\hline Wk 8 & 40 & + & ++ & ++ & ++ \\
\hline Wk 11 & 37 & + & +++ & + & + \\
\hline
\end{tabular}

Inflammatory reaction: - no visible signs of inflammatory response, + mild inflammatory response, ++ significant inflammatory reaction, +++ severe inflammatory infiltrates. Organization of graft: - connective tissue organization absent or slight, + isolated fibroblasts within graft. Foreign body reaction with multinuclear giant cells: - no foreign body reaction, + mild foreign body reaction, ++ significant foreign body reaction, +++ extensive foreign body reaction.

tions and in-line with earlier studies [13].

The reaction at 2 weeks with some inflammation and overlying adhesions is normal and has been documented in studies of other biologic materials. In the case of the fish skin graft, the inflammation however subsidizes until at 11 weeks, when there is no evidence of inflammation or any adhesions. This is in contrast to findings by Neulen et al., were three groups demonstrated a high ration of adhesions at 24 weeks. The materials tested by Neulen et al. were a semisynthetic collagen bilayer ("Duragen") and an autologous periosteum repair group. The incidence of macroscopic adhesions (soft or considerable) was $80 \%$, 33\% and $25 \%$ respectively [14]. Both the sheep that were sacrificed at 5 and 8 weeks had moderate to minor adhesions respectively. As a result of not securing the bony flaps with a plate and screw, both of those sheep had significant bony intrusion into the skull, with subsequent pressure onto the cerebral surfaces possibly resulting in adhesion being noted between the brain and the intrusive bone. In a larger study this needs to be taken into account and the bony flap fixed with a plate and screw.

\subsection{Histology}

The histological changes documented an initial acute inflammatory response with a foreign body reaction at 2 weeks postoperatively. The foreign body reaction then diminished and finally led to the formation of a mature neodura merging smoothly with the surrounding native dura at 11 weeks. Concluding that the initial inflammatory response was followed by vascular in-growth leading to fibroblast migration into the acellular fish skin and finally the deposition of new tissue integrating seamlessly with the surrounding native dura.

The findings are in keeping with the results from Knopp et al., who reported similar results using sheep as an experimental model to evaluate a new equine collagen biomatrix for dura replacement [13].

Inflammatory changes in the subarachnoid and subdural space were regressing from week 5 postoperatively. Focal lymphoid infiltrates were identified directly below the graft site at 8 weeks after operation-likely because of minute iatrogenic injury to the arachnoidea during operation.

The findings in this small pilot study are favorable when compared with the findings of specific previous animals trials, were some of the tested products demonstrated significant osseous metaplasia and significant fibrosis [1]. Neither osseous metaplasia nor fibrosis was demonstrated in the study of the acellular fish skin.

Neulens et al. published the results of a porcine model with lymphocytic infiltration at 4 weeks as well as suture granulomas. This was then shown to have a diminishing inflammatory response at 24 weeks [14].

An optimal experimental design is often a compromise between the number of animals on the one hand, and the ethical and economic costs of performing the study on the other hand. The positive findings in this initial study argue for the continued use of sheep in a larger study on the efficacy of acellular fish skin in dura depair.

\subsection{Neuroimaging}

In none of the subjects was there evidence of CSF leak, which is in-line with the necropsy findings, where the fish skin had integrated into the surrounding dura mater. Overall the results demonstrated diminishing signal 
abnormality and post contrast enhancement from 2 to 11 weeks with the final images at 11 weeks demonstrating very little if any abnormal findings.

A study in a canine beagle model by Asher et al. explored the use of a dural sealant. MRI scans were performed at day 3 and then monthly afterwards. The first scan demonstrated hyperintensivity in the T2 and T1 with contrast considered to be the result of postoperative oedema. This hyperintensity had subsided at the one month scan in both the active and control groups. This study also noted minor displacements in relation to the bony flaps as in our study. On follow up MRI from one month onward there were visible contrast enhancements related to the treatment article only dissipitating at 5 and 6 months [11]. In our study the contrast enhancement had dissipitated sooner, or at 11 weeks ( $<3$ months).

As the bony flap was not secured with a plate and screw, there were significant changes between the individual sheep in the position of the bony flap, in particular the sheep at 5 and 8 weeks. Those sheep had significant intrusions of the bony flap into the calvarium, with some associated pressure onto the cerebral surface. The interpreting radiologist concluded that this intrusion resulted in some underlying cerebral edema and in the case of the sheep at 5 weeks a small intracerebral hemorrhage. This needs to be taken into account when further test will be done by fixing the bony flap with a plate and screws postoperatively.

We are not aware of any other study performing regular MRIs after dura repair in a large animal model studying biologic grafts.

\section{Conclusion}

Kerecis Omega3 Dura ${ }^{\mathrm{TM}}$ performed safely and effectively in this pilot study and needs to be further assessed in a larger scale animal study.

\section{Acknowledgements}

Keldur research facility staff.

\section{References}

[1] Barbolt, T.A., Odin, M., Léger, M., Kangas, L., Holste, J. and Liu, S.H. (2001) Biocompatibility Evaluation of Dura Mater Substitutes in an Animal Model. Neurological Research, 23, 813-820. http://dx.doi.org/10.1179/016164101101199405

[2] Cappabianca, P., Esposito, F., Cavallo, L.M., Messina, A., Solari, D., di Somma, L.G.M. and de Divitiis, E. (2006) Use of Equine Collagen Foil as Dura Mater Substitute in Endoscopic Endonasal Transsphenoidal Surgery. Surgical Neurology, 65, 144-148; Discussion 149. http://dx.doi.org/10.1016/j.surneu.2005.08.023

[3] Cavallo, L.M., Solari, D., Somma, T., Somma, A.Di, Chiaramonte, C. and Cappabianca, P. (2013) Use of Equine Pericardium Sheet (LYOMESH ${ }^{\circledR}$ ) as Dura Mater Substitute in Endoscopic Endonasal Transsphenoidal Surgery. Università degli Studi di Salerno, 7, 23-28.

[4] Costa, B.S., Cavalcanti-Mendes, G.D.A., Abreu, M.S.De. and Sousa, A.A.De. (2011) Clinical Experience with a Novel Bovine Collagen Dura Mater Substitute. Arquivos de Neuro-Psiquiatria, 69, 217-220. http://dx.doi.org/10.1590/S0004-282X2011000200015

[5] Esposito, F., Cappabianca, P., Fusco, M., Cavallo, L.M., Bani, G.G., Biroli, F. and Signorelli, A. (2008) Collagen-Only Biomatrix as a Novel Dural Substitute: Examination of the Efficacy, Safety and Outcome: Clinical Experience on a Series of 208 Patients. Clinical Neurology and Neurosurgery, 110, 343-351. http://dx.doi.org/10.1016/j.clineuro.2007.12.016

[6] Williams, L.E., Vannemreddy, P.S., Watson, K.S. and Slavin, K.V. (2013) The Need in Dural Graft Suturing in Chiari I Malformation Decompression: A Prospective, Single-Blind, Randomized Trial Comparing Sutured and Sutureless Duraplasty Materials. Surgical Neurology International, 4, 26. http://dx.doi.org/10.4103/2152-7806.107904

[7] Warren, W.L., Medary, M.B., Dureza, C.D., Bellotte, J.B., Flannagan, P.P., Oh, M.Y. and Fukushima, T. (2000) Dural Repair Using Acellular Human Dermis: Experience with 200 Cases: Technique Assessment. Neurosurgery, 46. http://dx.doi.org/10.1097/00006123-200006000-00020

[8] Esmonde, T., Lueck, C.J., Symon, L., Duchen, L.W. and Will, R.G. (1993) Creutzfeldt-Jakob Disease and Lyophilised Dura Mater Grafts: Report of Two Cases. Journal of Neurology, Neurosurgery \& Psychiatry, 56, 999-1000. http://dx.doi.org/10.1136/jnnp.56.9.999

[9] Hoshi, K., Yoshino, H., Urata, J., Nakamura, Y., Yanagawa, H. and Sato, T. (2000) Creutzfeldt-Jakob Disease Associated with Cadaveric Dura Mater Grafts in Japan. Neurology, 55, 718-721. http://dx.doi.org/10.1212/WNL.55.5.718 
[10] Sekhar, L.N. and Mai, J.C. (2013) Dural Repair after Craniotomy and the Use of Dural Substitutes and Dural Sealants. World Neurosurgery, 79, 440-442. http://dx.doi.org/10.1016/j.wneu.2011.12.062

[11] Asher, A.L., Carnahan, M.A., Boyd, R.B., Adams, E.L. and Butt, M.T. (2010) Adherus Dural and Spinal Sealant as Adjuncts to Sutured Dural Repair in a Canine Lumbar Durotomy Repair Model. HyperBranch Medical Technology, Inc., Durham.

[12] Çetin, B., Şengül, G., Tüzün, Y., Gündoğdu, C., Kadioğlu, H.H. and Aydin, İ.H. (2006) Suitability of Collagen Matrix as a Dural Graft in the Repair of Experimental Posterior Fossa Dura Mater Defects. Turkish Neurosurgery, 16, 9-13.

[13] Knopp, U., Christmann, F., Reusche, E. and Sepehrnia, A. (2005) A New Collagen Biomatrix of Equine Origin versus a Cadaveric Dura Graft for the Repair of Dural Defects-A Comparative Animal Experimental Study. Acta Neurochirurgica, 147, 877-887. http://dx.doi.org/10.1007/s00701-005-0552-0

[14] Neulen, A., Gutenberg, A., Takács, I., Wéber, G., Wegmann, J., Schulz-Schaeffer, W. and Giese, A. (2011) Evaluation of Efficacy and Biocompatibility of a Novel Semisynthetic Collagen Matrix as a Dural Onlay Graft in a Large Animal Model. Acta Neurochirurgica, 153, 2241-2250. http://dx.doi.org/10.1007/s00701-011-1059-5 